Volume 7, No. 2

April - June 2021

www.jrmi.rmi.edu.pk

Submitted

Accepted

May 29, 2021

Author Information

From: Rehman Medical Institute, Peshawar, Khyber Pakhtunkhwa, Pakistan

Dr. Eemaz Nathaniel

House Officer

(Corresponding Author)

Email:

eemaz.nathaniel@rmi.edu.pk

Dr. Aimen James

House Officer

Citation: Nathaniel E, James

A. Dementia: need for studies in the new era. [Editorial]. $\mathrm{J}$ Rehman Med Inst. 2021;7(2):1-2.

\title{
Dementia: need for studies in the new era
}

Eemaz Nathaniel, Aimen James

\section{ABSTRACT}

Current insights into the structure and function of the human brain has brought about revolutionary changes in concepts about normal and abnormal conditions affecting it. An age-old issue is that of Dementia, previously ignored as an inevitable consequence of aging or mental disorder, that can now be studied in greater depth and perhaps manged more efficiently in light of new research.

The authors declared no conflict of interest. All authors contributed substantially to the write-up of the editorial and agreed to be accountable for all aspects of the work.

\section{INTRODUCTION}

Dementia according to ICD -10 classification of mental and behavioural disorder (WHO 1992) is a syndrome occurring due to brain diseases which is usually chronic and progressive. ${ }^{1}$

It's a Latin word that has been coined by Philippe Pinel in $1797 .{ }^{2}$

Dementia is not a single disease but perhaps a clinical syndrome with many causes, making the person more prone/vulnerable both physically and mentally. It a general term used for impairment of higher cortical functions such as learning, thinking, language, memory, calculation, comprehension, and judgment.

Dementia is a global health issue that usually affects the older population of age above 60 years with a prevalence of about $7 \%$ of individual above 65 years of age, ${ }^{3}$ it is estimated that the number of dementia cases worldwide is about 44 million and it will increase to double every year. ${ }^{1}$

The clinical diagnosis of dementia is difficult because the decrease in cognitive function in dementia is due to many causes of which Alzheimer's diseases are the most common (AD; 50-75\%), followed by Vascular dementia (VAD; 20\%), Dementia With Lewy Body (DLB; 5\%) Frontotemporal Lobar Dementia (FTLD; 5\%), to lesser extend multiple sclerosis, Creutzfeldt-Jacob disease, HIV/AIDS (3\%) are also involved. ${ }^{4}$

Known risk factors for dementia are the following:

1) Age: chances of having this syndrome increases with age (risk doubles after $65 \mathrm{yrs}$ ).

2) Gender: females are more prone to this disease. $^{5}$

3) Genetics: plays a special role in Alzheimer disease. $^{6}$
The main goals of treatment are to stop the progression of disease, give symptomatic relief, and stabilize the patient. It includes both pharmacological and non-pharmacological approaches.

A) Pharmacological treatment is further divided into:

- AD (Alzheimer dementia) treatment: in which Acetylcholinesterase inhibitor and N Methyl D Aspartic Acid (NMDA) is used; ${ }^{7}$ and

- NON-AD dementia treatment includes Donepezil, Rivastigmine and Galantamine. Several clinical trials have shown that Antihypertensive can help to prevent VAD (vascular dementia). ${ }^{8}$

B) Nonpharmacological treatment includes cognitive-based intervention, psychosocial therapies, and physical activity.

Why the level of care of dementia in Pakistan is deficient?

1) Do we know the exact aetiology of dementia?

2) How are our health services?

3) What is the amount of data collection or research done on dementia?

The exact aetiology and pathophysiology of dementia have not been fully understood till now, which makes it difficult for the health care workers to know what they are dealing with. Mostly the caretakers is family members because health care specialist are only available through private channels accessible only by wealthy. ${ }^{9}$ Overall, the basic economic rights of older people in our country are not well defined. Meanwhile, research conducted in Pakistan about dementia is very limited, which makes it even more difficult to understand this disease in our community. ${ }^{10}$

The studies that have been conducted about knowledge of dementia showed that only a fair to moderate level of information about this disease is present in the participants. The most common general misconception about dementia in the general public is that it is normal to suffer from it in older age, this is where their level of knowledge can be assessed. ${ }^{11}$

Therefore, it is important to conduct enough research among the middle and low-income countries to prevent the occurrence of it. 


\section{REFERENCES}

1. Dening T, Sandilyan MB. Dementia: definitions and types. Nurs Stand 2015;29(37):37-42.

2. Boller F, Forbes MM. History of dementia and dementia in history: An overview. J. Neurol. Sci. 1998;158(2):125-33.

3. Gale SA, Acar D, Daffner KR. Dementia. Am J Med. 2018;131(10):1161-9.

4. Whatmough C.E. Dementia. In: Armstrong C., Morrow L. (eds). Handbook of Medical Neuropsychology. Cham, Switzerland: Springer; 2019. Available from: https://doi.org/10.1007/978-3-030-148959_17.

5. WHO. Dementia. [Fact Sheet] Accessed May 10, 2021. Available from: https://www.who.int/news-room/factsheets/detail/dementia.

6. National Institute on Aging. Alzheimer's Disease Genetics Fact Sheet. Accessed May 10, 2021. Available from: https://www.nia.nih.gov/health/alzheimersdisease-genetics-fact-sheet.

7. Grand JHG, Caspar S, MacDonald SWS Clinical features and multidisciplinary approaches to dementia care. J Multidisci Healthc. 2011;4(May 2011):125-47.

8. In't Veld BA, Ruitenberg A, Hofman A, Stricker BHC, Breteler MMB. Antihypertensive drugs and incidence of dementia: The Rotterdam Study. Neurobiol Aging. 2001;22(3):407-12.
9. Balouch S, Zaidi A, Farina N, Willis R. Dementia awareness, beliefs and barriers among family caregivers in Pakistan. Dementia. 2020:1-20.

10. Khan Q. Global perspectives. Neurology. 2014;83(22):2091-2. Accessed May 10, 2021. Available from: https://pubmed.ncbi.nlm.nih.gov/25422399

11. Cahill S, Pierce M, Werner P, Darley A, Bobersky A. A systematic review of the public's knowledge and understanding of Alzheimer's Disease and Dementia. Alzheimer Dis Assoc Disord 2015;29(3):255-75. 